\title{
Ultrabright femtosecond electron sources: perspectives and challenges towards the study of structural dynamics in labile systems
}

\author{
Meng Gao ${ }^{\mathrm{a}, \mathrm{b}}$, H. Jean-Ruel ${ }^{\mathrm{a}, \mathrm{b}}$, Cheng Lu ${ }^{\mathrm{a}}$, L. C. Liu ${ }^{\mathrm{a}, \mathrm{b}}$, A. Marx ${ }^{\mathrm{b}}$, R. R. Cooney ${ }^{\mathrm{a}}$, Y. Jiang ${ }^{\mathrm{a}, \mathrm{b}}$, G. H. \\ Kassier $^{\mathrm{a}, \mathrm{b}}$, G. Moriena ${ }^{\mathrm{a}, \mathrm{b}}$, G. Sciaini ${ }^{\mathrm{a}, \mathrm{b},{ }^{*}}$ and R. J. D. Miller ${ }^{\mathrm{a}, \mathrm{b}}$ \\ ${ }^{\mathrm{a}}$ Departments of Chemistry and Physics, 80 St. George Street, University of Toronto, Toronto, \\ Ontario, M5S 3H6, Canada \\ ${ }^{\mathrm{b}}$ Max Planck Institute for the Structure and Dynamics of Matter, and Hamburg Centre for Ultrafast \\ Imaging, Luruper Chaussee 149, 22761 Hamburg, Germany. \\ *Present address: Department of Chemistry, 200 University Ave. W, University of Waterloo, \\ Ontario, N2L 3G1, Canada. \\ Emails: gsciaini@uwaterloo.ca and dwayne.miller@mpsd.mpg.de
}

\begin{abstract}
The advances made in femtosecond electron sources over the last thirty years have been remarkable. In particular, the development of ultrabright femtosecond electron sources has made possible the observation of molecular motion in labile organic materials and it is paving the way towards the study of complex protein systems. The principle of radio frequency (RF) rebunching cavities for the compression of ultrabright electron pulses is presented, alongside with a recent demonstration of its capabilities in capturing the relevant photoinduced dynamics in weakly scattering organic systems. Organic and biological systems can easily decompose or lose crystallinity as a consequence of cumulative heating effects or the formation of side reaction photoproducts. Hence, source brightness plays a crucial role in achieving sufficient signal-to-noise ratio before degradation effects become noticeable on the structural properties of the material. The current brightness of electron sources in addition to the high scattering cross section of keV-MeV electrons have made femtosecond electron diffraction a powerful tool for the study of materials composed by low-Z elements.
\end{abstract}

Keywords: Ultrafast electron diffraction, structural dynamics, molecular motion.

\section{INTRODUCTION}

The progress in the development of ultrafast electron sources for time-resolved diffraction has been tremendous. We are now able to reach the spatial (sub-Angstrom) and temporal (sub-picosecond) resolutions required to literally see atoms move ${ }^{1,2}$. The trick relies on generating short enough pulses of electrons with the appropriate de Broglie wavelength to diffract off the underlying nuclear configuration. The technique is known as ultrafast electron diffraction (UED), an unconventional pump-probe approach in which the femtosecond (fs) optical probe pulse has been replaced by an ultrashort "structure-sensitive" electron burst. UED has been applied to a variety of crystalline samples and provided detailed information about photoinduced structural processes. However, its application to labile organic materials has only been recently realized ${ }^{3,4}$. Source brightness has been one of the most challenging tasks to extend UED to effectively monitor molecular motion in labile organic systems. Organic crystals possess low melting points, poor heat conduction, and are comprised of weak scattering centers. All these facts have made the observation of ultrafast structural dynamics in organic samples a real challenge. In UED a femtosecond optical "pump" pulse initiates the dynamics while the structural changes are monitored by time-delayed diffraction patterns. Cumulative heating and/or photo-degradation effects from the laser pump pulse are finally responsible of ruining the sample crystallinity and ultimately limiting its exposure time. Studies of organic systems therefore required the development of an ultrabright fs-electron source able to circumvent the temporal deterioration of the electron bunch caused by space-charge effects ${ }^{5-7}$. Given the charged nature of the electron, Coulomb repulsion or space-charge effects broaden the temporal resolution by kicking electrons within the bunch apart so that the electron pulse spreads as it propagates towards the sample. It is the electron pulse duration at the sample position what finally determines the temporal resolution. This article will outline the development, characterization, and application of a novel electron compression scheme based on a radio frequency (RF) cavity, which

Ultrafast Nonlinear Imaging and Spectroscopy II, edited by Zhiwen Liu, lam Choon Khoo,

Demetri Psaltis, Proc of SPIE Vol. 9198, 91980R - C) 2014 SPIE

CCC code: $0277-786 \mathrm{X} / 14 / \$ 18 \cdot$ doi: $10.1117 / 12.2062888$ 
has allowed us to resolve the key molecular displacements involved in a photoinduced insulator-to-metal phase transition and a ring-closing reaction occurring in pure organic crystalline samples. The use of a time-stamping technique for fs electron pulses is discussed as a possibility to improve the overall instrument response function down to the sub-100 fs time domain. Finally, a newer design based on ultra-compact electron guns is presented.

\section{RF ELECTRON PULSE COMPRESSION}

The scheme of the hybrid DC-RF electron gun built at the University of Toronto is shown in figure 1 . The concept relies on the linear momentum-space distribution that sub-relativistic electrons develop within a dense bunch after several centimeters of propagation ${ }^{8}$. The effect is illustrated in figure 1, lower panels.

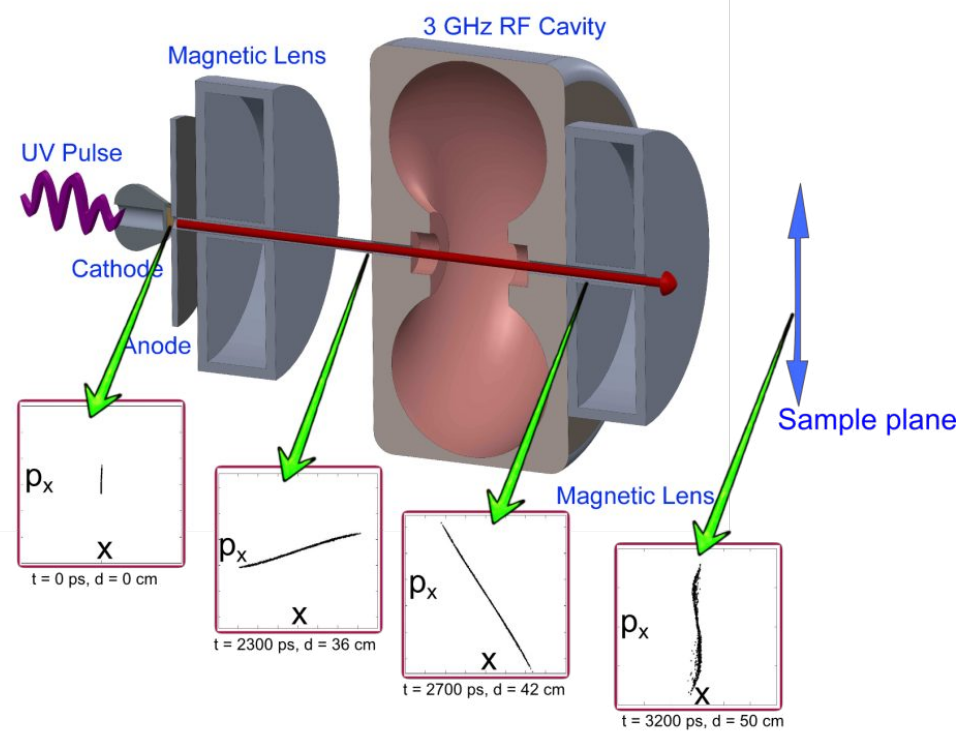

Figure 1. Hybrid 95kV DC-RF electron gun built at the University of Toronto. Upper panel: 3D CAD design adapted from reference 6, C Copyright 2014, The Optical Society. Lower insets: momentum-space electron distribution within the burst at birth, before and after the RF pillbox. N-body calculations were performed using The General Particle Tracking code ${ }^{9}$.

Analogous to pulse compression in optics, where a linear chirped pulse can be compressed through the implementation of an array of dispersive media such as gratings or prisms, charged particles distributed in a linear fashion in momentumspace can reverse their phase through the application of a suitable time-dependent electric field. The simplest electron optic that can produce this electric field is a standard $\mathrm{TM}_{010} \mathrm{RF}$ pillbox design that employs a resonant frequency of $\sim 3$ $\mathrm{GHz}$ to benefit from existing technology. This RF cavity provides a time varying axial electric field that is uniform along the cavity $z$-axis (red arrow in figure 1) and varies in time as $E_{z}(z, t)=E_{0}(z) \cos (2 \pi f t+\phi)$; where $f$ is the resonant frequency of the cavity and $\phi$ a phase that is chosen such that $E_{z} \equiv 0$ when the centroid of the electron bunch is at the center of the pill lens. This value of $\phi$ assures that there is no overall gain or loss of the average electron pulse's kinetic energy. The electron pulses are generated via the photoelectric effect by back illuminating a thin film gold photocathode with 50-fs, 270-nm optical pulses. Electrons are rapidly accelerated by a $95 \mathrm{kV}$ DC electric field towards a disk anode with a small aperture of typically $200 \mu \mathrm{m}$. A magnetic lens collimates the stripped electron bunch before entering the RF cavity. The electron bunch at the entrance of the RF cavity is positively chirped. When the electron pulse reaches the center of the RF pillbox, the electric field changes its direction in order to retard the electrons at the front of the pulse while the electrons at back are relatively kicked in the forward direction. The overall effect results in the reversion of the momentum-position distribution of the electrons within bunch. At the exit of the RF cavity the electron bunch is negatively chirped. The sample position is therefore chosen to coincide with the temporal focus of the RF pill lens. UED systems with RF electron pulse compression are delivering an instrument temporal response (IRF) of 300-500 fs (FWHM), which is currently limited by RF synchronization noise. 


\section{INSTRUMENT CHARACTERIZATION}

We implemented enhanced ponderomotive scattering to determine the IRF of the aforementioned setup. The experimental arrangement is shown in figure 2. Two counter-propagating optical pulses generate a transient optical grating that acts as a scattering medium for the electrons. The electrons experience a ponderomotive force $(F)$ that is proportional to the gradient of the laser intensity $(\nabla I)$.

$$
F(\boldsymbol{r}, t)=\frac{-\mathrm{e}^{2} \lambda^{2}}{8 \pi^{2} \mathrm{~m}_{\mathrm{e}} \varepsilon_{0} \mathrm{c}^{3}} \nabla I(\boldsymbol{r}, t)
$$

where $e$ and $m_{e}$ are the charge and mass of the electron, respectively, $\lambda$ is the wavelength of the laser, $\varepsilon_{0}$ is the permittivity of vacuum, and $\mathrm{c}$ is the speed of light. The measured signal is defined as $\mathrm{S}(\tau)=\int|X| D_{\tau}(X, Y) d X d Y(2)$, where $X$ and $Y$ are the horizontal and vertical coordinates on the detector screen (origin at electron beam center) and $D_{\tau}(X, Y)$ is the image intensity (proportional to the electron density) at a particular position on the screen, and a given time delay $\tau$. Under this definition the temporal variance of the signal trace $\left(\sigma_{\mathrm{S}}^{2}\right)$ can be written ${ }^{10}$ as $\sigma_{\mathrm{S}}^{2}=\sigma_{e-t}^{2}+\sigma_{t}^{2}+$ $\sigma_{w}^{2} / v_{e}^{2}(3)$, where $\sigma_{e-t}$ reflects the IRF, a convolution between the electron pulse length and its arrival timing jitter, $\sigma_{t}^{2}$ is temporal variance of optical pulses, $\sigma_{w}^{2}$ is the spatial variance of the laser beam size at the intersection, and $v_{e}$ is the velocity of $95 \mathrm{keV}$-electrons with respect to the lab frame. From the variance of the signal trace shown in figure 2 (c) we obtained $\sigma_{e-t}=183 \pm 32 \mathrm{fs}$, which assuming a Gaussian distribution provides an IRF of $430 \pm 75 \mathrm{fs}$ at full-width-halfmaximum (FWHM).

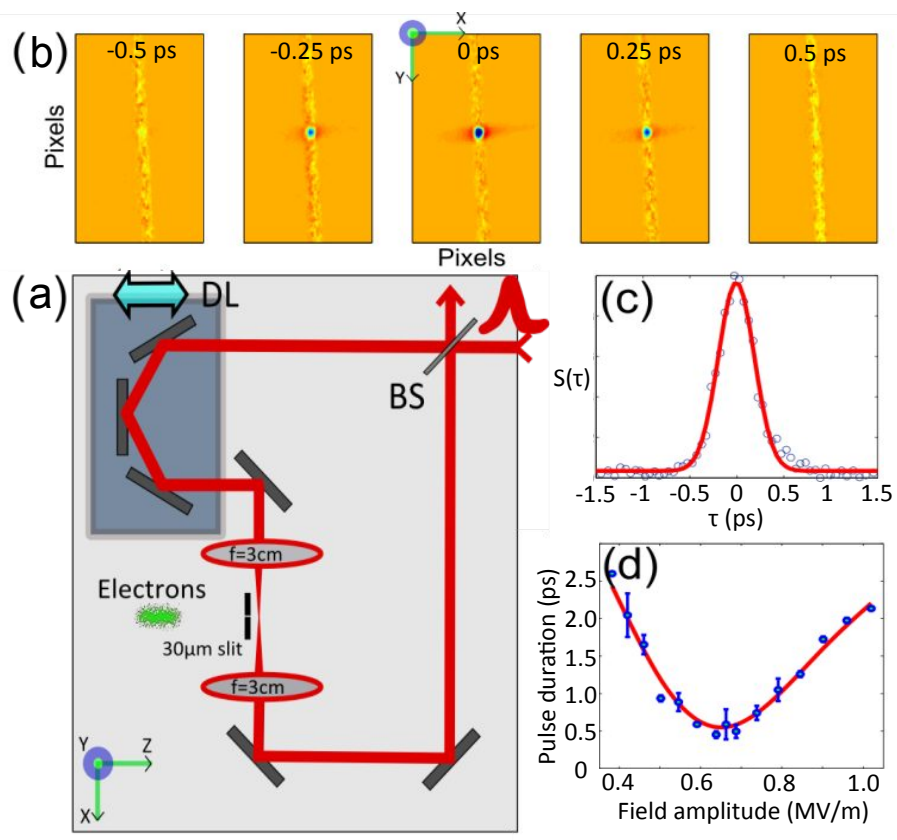

Figure 2. Ponderomotive measurements. (a) Layout of the optical arrangement for enhanced ponderomotive signal. A beam splitter (BS) is used to generate two counter-propagating laser pulses that meet at the focal point of two short focal length lenses. The interaction between the laser fields generates a transient standing wave that is used to scatter electrons via the ponderomotive force. A motorized delay line (DL) is used to assure equality in the optical path lengths of the two counterpropagating arms. This is achieved by maximizing the scattering effect. Another delay stage placed in the electron beam path is used to set the relative time delay $(\tau)$ between the formation of the transient optical grating and the arrival time of the electron bunch. A slit is used to strip the electron beam size in the $x$-direction to about $30 \mu \mathrm{m}$ to better match the lateral size of the optical standing wave and make the ponderomotive effect visible. (b) Each image shows a colored map of the difference between the detected electron beam intensity with and without the presence of laser field for different $\tau$. Blue color represents a negative change in the electron density and the red color represents a positive change. The scattering effect produced by the ponderomotive force is clearly visible. The slit used to strip the electron beam appears slightly tilted due to the detector orientation. The RF field amplitude used for the measurements shown in panels (b) and (c) was $0.67 \mathrm{MV} / \mathrm{m}$. (c) Electron bunch-optical grating cross-correlation signal. The scattering signal (S) is calculated at each $\tau$ according to eq. 2, see main text. (d) Measured IRF (FWHM) as a function of the RF field amplitude. Optimal compression for this particular setup configuration was achieved at approximately $0.67 \mathrm{MV} / \mathrm{m}$. Images were adapted with permission from reference 6, (C) Copyright 2014, The Optical Society. 


\section{MOLECULAR MOTION IN LABILE ORGANIC CRYSTALS}

Throughout the implementation of the aforementioned ultrabright femtosecond electron source we have been able to observe molecules in motion and the formation of transient intermediate structures (TISs) in two different organic crystals. Organic samples present poor heat conduction and low melting points that make them sensitive to cumulative heating effects, which impart low damage thresholds. In addition, cumulative heating effects can mask the dynamics and induce the formation of side photoproducts, which can finally ruin the crystalline structure of the material. Source brightness and very low repetition rates are therefore crucial to succeed in the study of labile molecular systems by UED. Figure 3 shows some recent results obtained for $(\mathrm{EDO}-\mathrm{TTF})_{2} \mathrm{PF}_{6}$ (upper panels, $(\mathrm{a}, \mathrm{c})$ ) and diarylethene (lower panels, $(b, d)$ ). EDO-TTF stands for ethylenedioxytetrathiafulvalene and the diarylethene derivative studied was 1,2-bis(2,4dimethyl-5-phenyl-3-thienyl)perfluorocyclopentene. Panels $(a, b)$ show characteristic electron diffraction patterns while panels (c, d) present the photoinduced relative intensity changes obtained for some selected Bragg spots. The formation of TISs has been observed for both compounds undergoing two very different photoinduced processes, an insulator-tometal phase transition in the case of $(\mathrm{EDO}-\mathrm{TTF})_{2} \mathrm{PF}_{6}$ and a ring-closing reaction in diarylethene. The difference in signal-to-noise ratio (SNR) between the traces shown in figure 3 (c) and the trace in figure 3 (d) is a consequence of the very limited number of $(\sim 300)$ of repeatable photoexcitation cycles per sample in diarylethene and the significantly lower repetition rate $(\sim 0.1 \mathrm{~Hz})$ imposed by the thermal irreversibility of ring-closing reaction which required every fs uv pump pulses to be followed by continuous visible illumination for cycloreversion. The generation of side reaction photoproducts that play a significant role in the crystalline structure of diarylethene is very evident and illustrates the need of ultrabright sources to access ultrafast structural dynamics in labile systems such as protein crystals. For details about the structural determination of the TISs, please refer to references 3,4 .
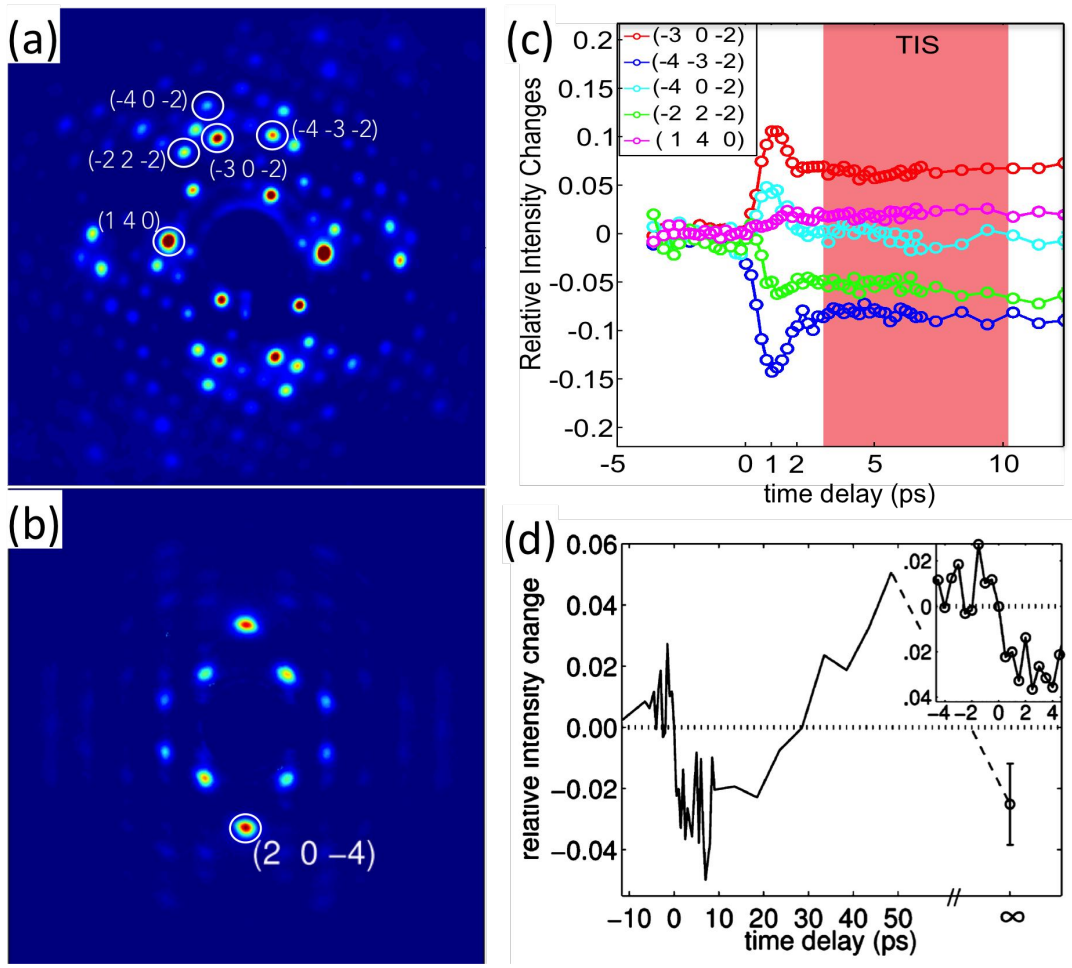

Figure 3. Electron diffraction patterns of (EDO-TTF) $)_{2} \mathrm{PF}_{6}$ (a) and diarylethene (b). Panels (c, d) show the observed photoinduced intensity changes of some selected Bragg peaks, which are indexed in panels $(a, b)$. Figures $(a, b)$ were adapted from reference 3. Figures (c d) were adapted with permission from reference 4. Copyright (2013) American Chemical Society. 


\section{TIME STAMPING OF FEMTOSECOND ELECTRON PULSES}

State-of-the-art particle tracking simulations have shown that it is possible to compress dense electron pulses down to sub-100 fs (FWHM) ${ }^{11,12}$. However, the main limitation in the temporal resolution achievable by RF electron pulse compression techniques arises from RF synchronization noise, i.e. phase and amplitude instabilities of the RF field. In general, phase noise with respect to the master clock is the dominant factor. Phase changes with respect to the ideal value (see above) results in an effective gain or loss of the average electron pulse's kinetic energy, and therefore translates into arrival time noise where each electron pulse reaches the sample at slightly different relative time delays with respect to the optical excitation or clocking pulse. We have demonstrated a technique based on time stamping, which can determine the electron pulse arrival time with a precision of sub-100 fs (FWHM). Several time stamping techniques have been applied to improve the temporal resolution in X-ray free electron lasers ${ }^{13-18}$. We have developed a time stamping tool based on a miniature ultra stable photo-triggered streak camera that is used to directly track the arrival time of each electron pulse in order to correct for the timing jitter in the radio frequency synchronization. Details about the streak camera can be found elsewhere ${ }^{19}$.

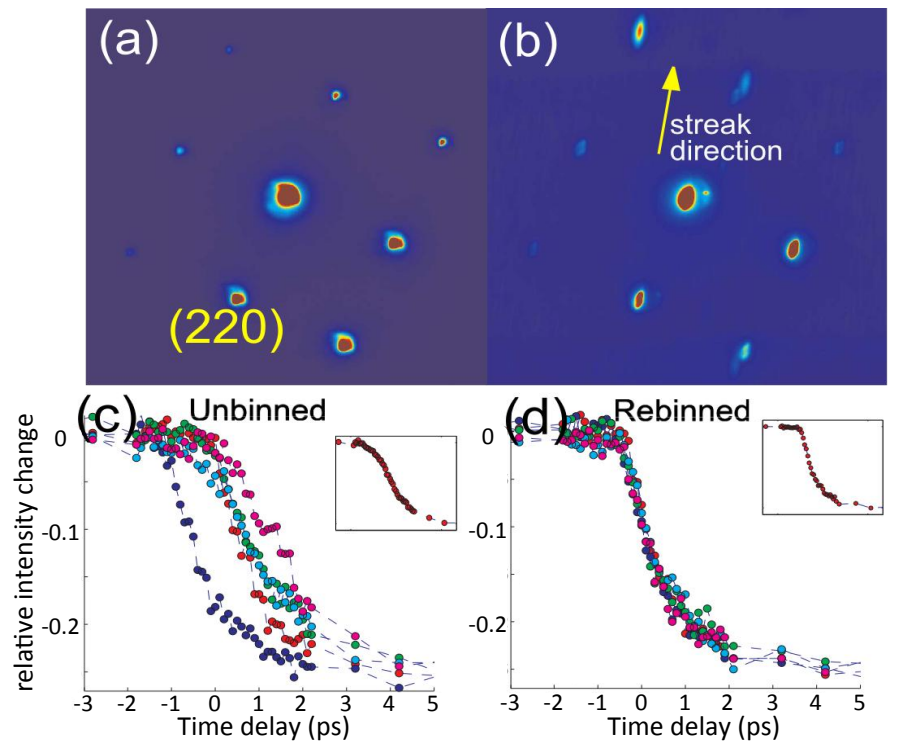

Figure 4. Panel (a) shows an averaged diffraction pattern of a (001)-oriented thin crystalline Si and panel (b) shows the an averaged diffraction pattern of the same sample but with the streak camera turned on. Panels (c) and (d) show the correction of timing by the stamping method, where the photoinduced relative intensity change of the (220) Bragg reflection is plotted as a function of time delay. Five traces were taken at $3 \mathrm{~h}$ time intervals, showing the effects of phase drift on the relative optical pump-electron probe time delay. Panel (d) presents the same data after correcting or re-binning each single-shot image using time stamping, i.e. from the displacement of the streaked diffraction patterns. The insets show the average of the 5 traces in (c) and (d), respectively. Images were adapted with permission from reference 20, C2013 AIP Publishing LLC.

The stamping procedure relies on determining the position of streaked diffraction peaks by performing a Gaussian fit or centroid analysis. The streak camera is set such that the displacement (camera on - off) of the diffraction pattern on the screen has a linear dependence on the relative time delay between the electron bunch and the optical pulse photoswitching the camera. This dependence is obtained through calibration, i.e. by measuring the displacement of the diffraction pattern as a function of the time delay. Figure 4 shows a proof of principle experiment performed on (001)oriented single crystalline thin film Si. A substantial drift in the electron pulse arrival time is evident in panel (c). The correction in timing by stamping and binning is shown in panel (d). Further improvements can be introduced depending on the type of detector used. For instance, detectors with a hollow center would allow performing the time stamping method post diffraction, using the zero-order (main) electron beam. This would avoid distorting the Bragg spots. 


\section{NEW COMPACT ELECTRON GUN DESIGNS}

Newer designs based on ultra-compact fs electron guns offer the most convenient and economic means to attain few 100 fs electron pulses carrying a maximal areal electron density similar to the one typically achievable by RF compression schemes $\left(\approx 5 \mathrm{e}^{-} / \mu \mathrm{m}^{2}\right)$. The most compact and simplest UED setup is comprised of a photocathode, an anode aperture and a cylindrical magnetic lens that is placed right after the sample. Figure 5 shows the calculated ${ }^{9}$ electron pulse duration as a function of the number of electrons (per pulse) that reach the sample plane for two different electron gun configurations (see figure's caption for details). The shortest cathode sample distance is obtained by placing the sample hosted by a TEM mesh straight on the anode aperture ${ }^{21}$. As can be inferred from the calculations the extracting DC electric field strength $(E)$ plays a major role. There is a great reduction in the electron pulse duration when going from $E$ $=10 \mathrm{MV} / \mathrm{m}$ (the typical field used in compact guns ${ }^{22}$ ) to $E=20 \mathrm{MV} / \mathrm{m}$. Reaching a field strength of $20 \mathrm{MV} / \mathrm{m}$ is challenging but possible $e^{23}$ and under this conditions such ultra-compact setup would enable sub-100 fs time resolution.
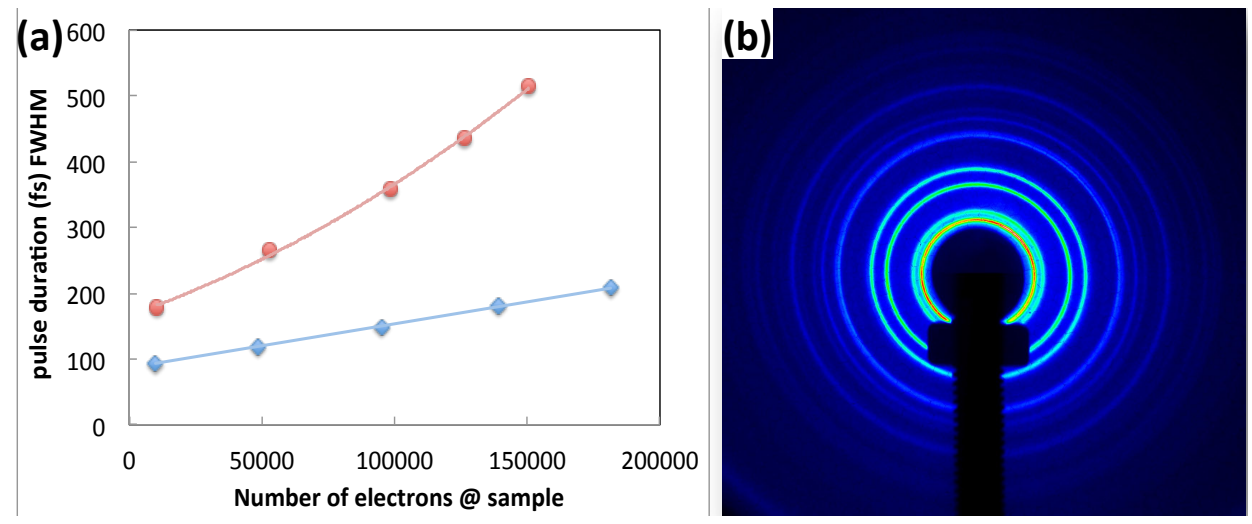

Figure 5. (a) Simulated UED setup performance for an ultra-compact DC electron gun. Blue trace: calculated electron pulse duration (FWHM) as a function of the number of electrons that reach the sample for a photocathode held at $-100 \mathrm{kV}$ with respect to an anode aperture $(200 \mu \mathrm{m}$ in diameter) located $5 \mathrm{~mm}$ downstream. The sample position was chosen to be $5 \mathrm{~mm}$ from the anode (10 mm from the cathode). The DC electric field between the cathode and anode plates is therefore $20 \mathrm{MV} / \mathrm{m}$. Red trace: same particle trace calculations performed for an electron gun with the same parameters but a cathode to anode distance of $10 \mathrm{~mm}$. Simulation parameters: lateral emission spot size at the cathode was assumed to be Gaussian, $200 \mu \mathrm{m}$ (FWHM). A Gaussian temporal profile of $50 \mathrm{fs}$ (FWHM) was considered. The electron emission was assumed to be isotropic with an initial energy spread of $0.2 \mathrm{eV}$. (b) Diffraction pattern obtained from a gold thin film by an ultra-compact 120-keV UED setup develop at Max Planck Institute for the Structure and Dynamics of Matter, Hamburg.

\section{REFERENCES}

[1] Miller, R. J. D., "Femtosecond Crystallography with Ultrabright Electrons and X-rays: Capturing Chemistry in Action," Science 343, 1108-1116 (2014).

[2] Sciaini, G. and Miller, R. J. D., "Femtosecond electron diffraction: heralding the era of atomically resolved dynamics," Rep. Prog. Phys. 74, 096101 (2011).

[3] Gao, M. et al., "Mapping molecular motions leading to charge delocalization with ultrabright electrons," Nature 496, 343-346 (2013).

[4] Jean-Ruel, H. et al., "Ring-Closing Reaction in Diarylethene Captured by Femtosecond Electron Crystallography," J. Phys. Chem. B 117, 15894-15902 (2013).

[5] Van Oudheusden, T. et al., "Compression of Subrelativistic Space-Charge-Dominated Electron Bunches for SingleShot Femtosecond Electron Diffraction," Phys. Rev. Lett. 105, 264801 (2010).

[6] Gao, M. et al., "Full characterization of RF compressed femtosecond electron pulses using ponderomotive scattering," Opt. Express 20, 12048-12058 (2012).

[7] Chatelain, R. P., Morrison, V. R., Godbout, C. and Siwick, B. J., "Ultrafast electron diffraction with radio-frequency compressed electron pulses," Appl. Phys. Lett. 101, 081901-081901-4 (2012). 
[8] Siwick, B. J., Dwyer, J. R., Jordan, R. E. and Miller, R. J. D., "Ultrafast electron optics: Propagation dynamics of femtosecond electron packets," J. Appl. Phys. 92, 1643-1648 (2002).

[9] Van der Geer, S. B. and de Loos, M. J., "Gen. Part. Tracer GPT Code," http://www.pulsar.nl/gpt.

[10] Hebeisen, C. T. et al., "Grating enhanced ponderomotive scattering for visualization and full characterization of femtosecond electron pulses," Opt. Express 16, 3334-3341 (2008).

[11] Van Oudheusden, T. et al., "Electron source concept for single-shot sub-100 fs electron diffraction in the $100 \mathrm{keV}$ range," J. Appl. Phys. 102, 093501-093501-8 (2007).

[12] Harb, M., "Investigating Photoinduced Structural Changes in Si using Femtosecond Electron Diffraction," PhD Thesis, University of Toronto (2009).

[13] Cavalieri, A. L. et al., "Clocking Femtosecond X Rays," Phys. Rev. Lett. 94, 114801 (2005).

[14] Krässig, B. et al., "A simple cross-correlation technique between infrared and hard x-ray pulses," Appl. Phys. Lett. 94, 171113-171113-3 (2009).

[15] Frühling, U. et al., "Single-shot terahertz-field-driven X-ray streak camera," Nat. Photonics 3, 523-528 (2009).

[16] Tavella, F., Stojanovic, N., Geloni, G. and Gensch, M., "Few-femtosecond timing at fourth-generation X-ray light sources," Nat. Photonics 5, 162-165 (2011).

[17] Grguraš, I. et al., "Ultrafast X-ray pulse characterization at free-electron lasers," Nat. Photonics 6, 852-857 (2012).

[18] Zhu, D. et al., "A single-shot transmissive spectrometer for hard x-ray free electron lasers," Appl. Phys. Lett. 101, 034103 (2012).

[19] Kassier, G. H. et al., "A compact streak camera for 150 fs time resolved measurement of bright pulses in ultrafast electron diffraction," Rev. Sci. Instrum. 81, 105103-105103-5 (2010).

[20] Gao, M., Jiang, Y., Kassier, G. H. and Miller, R. J. D., "Single shot time stamping of ultrabright radio frequency compressed electron pulses," Appl. Phys. Lett. 103, 033503 (2013).

[21] Gerbig, C., Morgenstern, S., Sarpe, C., Wollenhaupt, M. and Baumert, T., "Femtosecond Transmission Electron Diffraction on Single Crystalline Graphite," Res. Opt. Sci. IT3D.3 (Optical Society of America, 2012). doi:10.1364/ICUSD.2012.IT3D.3

[22] Hebeisen, C. T., "Generation, Characterization and Applications of Femtosecond Electron Pulses," PhD Thesis, University of Toronto (2009).

[23] Descoeudres, A., Ramsvik, T., Calatroni, S., Taborelli, M. and Wuensch, W., "Dc breakdown conditioning and breakdown rate of metals and metallic alloys under ultrahigh vacuum," Phys. Rev. Spec. Top. - Accel. Beams 12, 032001 (2009). 